\title{
On The Requirements for Quasi-Deterministic Radio Channel Models for Heterogeneous Networks
}

\author{
Kai Börner, Johannes Dommel, Stephan Jaeckel, Lars Thiele \\ Fraunhofer Institute for Telecommunications \\ Heinrich Hertz Institute \\ Einsteinufer 37, 10587 Berlin, Germany \\ Email: $\{$ kai.boerner, johannes.dommel, stephan.jaeckel, lars.thiele $\} @$ hhi.fraunhofer.de
}

\begin{abstract}
This paper introduces the key aspects of a new channel model based upon the Wireless World Initiative New Radio (WINNER)+ channel model for wireless channels in heterogeneous scenarios (e.g. indoor, outdoor and satellite scenarios). It consists of a collection of features created in the extended spatial channel model (SCME) and WINNER projects along with novel modeling approaches. The quasi deterministic radio channel generator (QuaDRiGa) ${ }^{1}$ is a geometry-based stochastic channel model (GSCM) which provides features to enable quasideterministic multi-link tracking of user equipments (UEs) movements in changing environments. The key aspects of QuaDRiGa are geo-correlated parameters maps, three dimensional antenna characteristics, a novel geometric polarization model, continuous drifting of small and large scale effects along UE movement, non-constant velocities as well as transitions between varying propagation scenarios (e.g. line of sight (LOS) and non line of sight (NLOS) conditions). The basic ideas and impacts of the additional features will be presented as well as the importance for future analyses.
\end{abstract}

Index Terms-WINNER, channel models, geometry-based channel modeling, correlation, polarization, antennas, satellite channels, MIMO, mobile radio, heterogeneous wireless networks

\section{INTRODUCTION}

Channel modeling is one of the key challenges in the design process of wireless communication systems. A common approach is to use a ray-based double-directional multi-link model such as the extended spatial channel model (SCME) [1] or the Wireless World Initiative New Radio (WINNER) [2] model. In this context, the wireless channel is modeled by a set of rays, having a direct connection or being scattered at obstacles in the surrounding environment denoted as line of sight (LOS) and non line of sight (NLOS) components, respectively. Each ray arrives at the receiver with a certain delay and power under a deterministic angle, yielding a multitap channel profile as depicted in Fig. 1. In this simplified figure only two rays are depicted.

The WINNER model, which is the evolution of the spatial channel model (SCM) and SCME model, is a geometry-based stochastic channel model (GSCM), where multiple rays are grouped into a scatterer cluster for a given multi-antenna configuration, as depicted in Fig. 1. The spatial distribution of the clusters, which specify the direction of the scattered

\footnotetext{
${ }^{1}$ QuaDRiGa is available at http://www.hhi.fraunhofer.de/quadriga
}

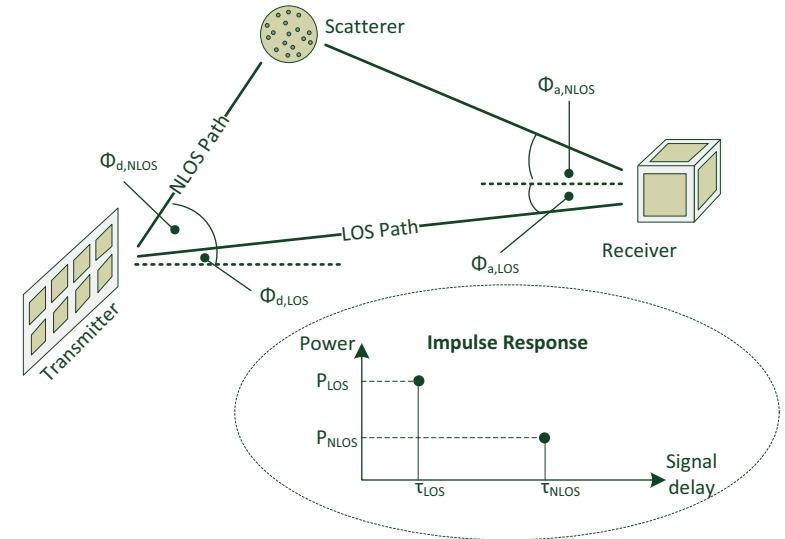

Fig. 1. Typical structure of geometry-based stochastic channel model, depicting the relation between LOS and NLOS components as well as the corresponding channel impulse response.

rays, are generated randomly. In contrast to strictly stochastic channel models, arbitrary antenna configurations and beam patterns are supported by GSCM. The parameters for generating the multiple-input multiple-output (MIMO) channel are extracted from measurements as recently summarized in [3]. One important aspect is the evolution of the channel over time. In the SCME and later on the WINNER model, time evolution of the channel is modeled on a short-term perspective as a drifting of the delays and departure/arrival angles. The large scale parameters (LSPs) such as path loss and shadow fading are kept constant over the observation period.

Here, the modeling approach of the quasi deterministic radio channel generator (QuaDRiGa) model diverges from the prior mentioned models by tracking all parameters deterministically according to a defined trajectory of the user equipment (UE). This property is especially valuable when considering long observation periods (in the order of several seconds) or higher velocities for the UEs. For these specific applications, the approaches SCME and WINNER models lack in accuracy of modeling the time evolution of the channel spanning several hundred wavelengths UE movement.

The quasi-deterministic time evolution along with other features of QuaDRiGa is of importance for the analysis of emerging heterogeneous network structures. Just to name some 


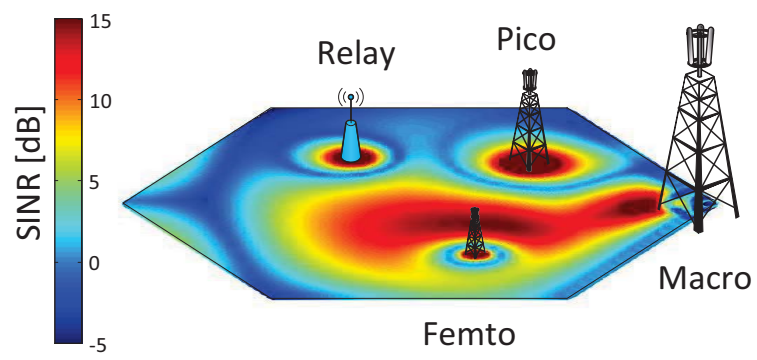

Fig. 2. Example for a wide-band SINR distribution shown as a cutout of a heterogeneous cellular network with full frequency reuse.

of the special use cases for heterogeneous scenarios:

- Transition between different channel scenarios, e.g. outdoor-to-indoor, urban macro

- Handover between cells on different hierarchical levels (longer observation periods)

- Channel prediction, especially for coordinated multi-point (CoMP) joint transmission

- Mobility profiles of UEs

- Spatially correlated channel characteristics for transmitters operating in direct vicinity

The main contribution of this paper is to identify the requirements and challenges for channel modeling of future heterogeneous networks. Further, we provide an overview on the QuaDRiGa channel model, which combines ideas of common GSCMs and includes novel features such as:

- Integration of enhanced time evolution features

- Improved large-scale parameter maps including geo-, cross- and inter-site correlation

- Novel 3-dimensional polarimetric antenna model

Fig. 2 shows an example of a wide-band signal to interference and noise ratio (SINR) coverage, i.e. usually referred as user geometry, taken from a large-scale heterogeneous cellular network operated at full frequency reuse.

The paper is structured as follows. Section II summarizes the main components of the proposed channel model QuaDRiGa. Section III provides insights for the necessity of different simulation complexities. The typical channel characteristics are given in section IV. The paper is than concluded in section $\mathrm{V}$.

\section{COMPONENTS OF QUADRiGA}

For a long period of time channel modeling was considered appropriately conducted using two dimensional propagation environment along with antenna characteristics valid only in the azimuth plane. Models like SCME [1] and WINNER1 followed this principle. In WINNER2 [2] an evolution towards an additional elevation component was made. All mentioned channel models that were implemented in WINNER were intended for short observation times in the order of several hundred milliseconds.
Simulations lasting over several seconds require special features especially with UEs traveling at high velocities. QuaDRiGa incorporates two levels at which the continuous time evolution is conducted. The first is the evolution between several snapshots which will be covered in II-E while the second level covers the evolution between segments of a track of a UE not sharing common propagation conditions which will be explained in II-E. Snapshots are points in time at which the channel is sampled. To understand how the time evolution works, the basic concepts of the QuaDRiGa model are explained in II-A, II-B, II-C II-D describing how a scenario is set up, how correlated parameter maps are generated, how antennas are modeled and how channel coefficients are generated in QuaDRiGa.

II-F provides information on how non-constant velocity profiles of UEs are taken into account for channel generation.

\section{A. Scenario Definition}

The first step to perform a simulation is to define a scenario by placing all transmitters, choosing a trajectory for all UEs, antenna configurations and propagation conditions. The propagation conditions consist of the carrier frequency, environment types (e.g. urban, rural, LOS, NLOS). Since QuaDRiGa is intended for long term observations, the environment types might change along the trajectory of the UEs. A method to keep track of propagation conditions is to generate parameter maps for every environment type for the whole area which is occupied by the trajectories of the UEs.

\section{B. Correlated Large Scale Parameter Maps}

In QuaDRiGa an environment type is characterized by seven properties also called LSPs: delay spread (DS), k-factor $(\mathrm{KF})$, shadow fading (SF) and the four angular spreads (ASs) for departure and arrival for elevation and azimuth direction (AsD, EsD, AsA, EsA). They are called LSP because they are valid for a range much greater than the wavelength of the wave emitted from the transmitter. Each of these LSPs follows a normal distribution which is being described by a median $\mu$ and a variance of $\sigma$. In order to describe the spacial correlation the autocorrelation distance $\lambda$ is needed as well as the cross-correlation between the LSPs. The significance of $\lambda$ is well known and explained in [4]. The autocorrelation is modeled by an exponential decay:

$$
\rho(d)=e^{-\frac{d}{\lambda}}
$$

In addition to the autocorrelation of the parameters, the cross-correlation coefficients between the LSPs and the crosscorrelations between different transmitter have to be taken into account which are independent of the scenarios types and are no part of the parameter sets. An exemplary run of curves for $\mathrm{SF}$ and rms DS is shown in Fig. 3 for macro and a micro base station along a continuous user track. The parameter maps provide a smooth evolution of the two LSPs over time. It can be seen that the autocorrelation distance of a macro base station is higher than the one of a micro base station. Here, a simple assumption is to set the inter-site correlation to 0.5 

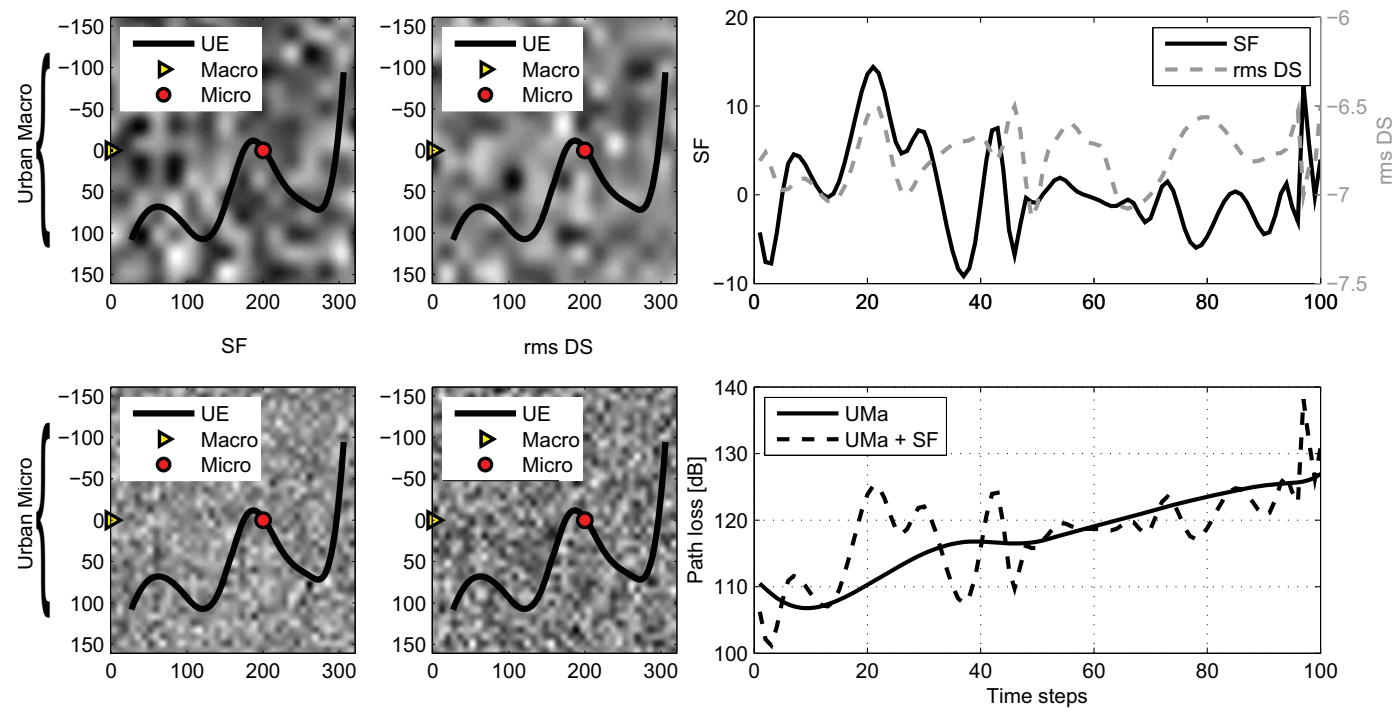

Fig. 3. Correlated large-scale parameter maps for shadow-fading and rms delay spread in a urban macro (top) and micro (bottom) environment.

and the intra-site correlation to 1 or employ a more complex method introduced in [5] where the relationship between the transmitters is considered.

\section{Antenna Modeling}

Realistic data models can be obtained by antenna vendors which provide the directivity for a vertical and horizontal cut. A satisfactory way to generate a three dimensional directivity from the two cuts was investigated in [6] and also suggested in [7]. Hence, the directivity is summed in logarithmic scale in elevation and azimuth direction also considering a lower bound for the maximum attenuation.

In addition to a realistic antenna pattern also a geometric polarization model is needed to accurately determine crosspolarization effects for along user trajectory. SCME completely lacked the ability to model polarization but could be extended by [8] to gain polarization capabilities. WINNER partially improved this polarization model. Both approaches have in common that the polarization properties could be statistically reproduced but not deterministically. We provide a new geometric model [9] of the 3D properties of the polarization. The model is based on elementary findings in the field of optics that can accurately predict the polarization state of a microwave LOS link.

\section{Channel Coefficients Calculation}

The generation of the channel coefficients is performed analogue to the WINNER2 [2] model, with the difference that the LOS component is assumed to be always present. Each scattering cluster (or propagation path) is modeled as a Dirac function in delay domain. The initial delays, cluster power, departure and arrival angles for each path are modeled as random variables, where the probability density function (pdf) and cross-correlation is a function of the correlated largescale parameter. The polarization for each path is calculated

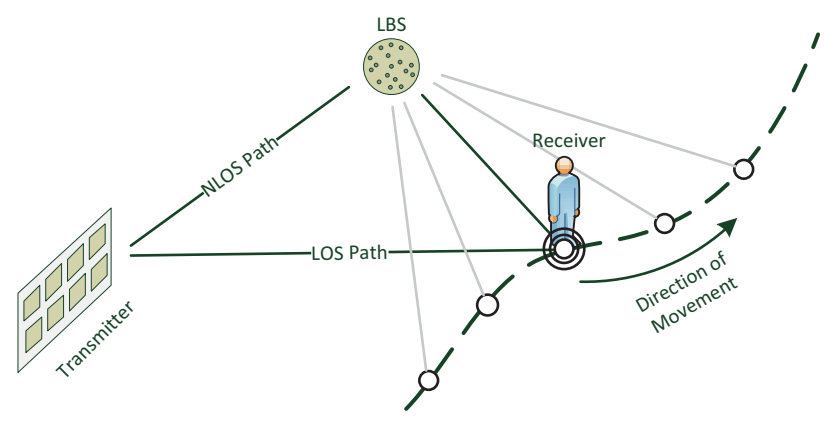

Fig. 4. Along with the knowledge of tx and rx position, delays and angles the NLOS and LOS path length can be determined.

in a geometric way for each antenna pair separately. Finally, the channel coefficients are calculated comprising the effect of the receiver and transmitter antenna pattern to each path.

\section{E. Time Evolution and Transitions Between Segments}

Time evolution in QuaDRiGa is understood as an evolution between snapshots within a segment on a user trajectory. As a matter of fact, the angle of departures (AoDs), angle of arrivals (AoAs), delays and phases are generated for a initial position per segment. The remaining snapshots in a segment have to be derived from the initial position exploiting the knowledge of the AoAs, AoDs and delays to the last bounce scatterers (LBSs) as shown in Fig. 4. The position of the LBSs can be determined by applying the cosine theorem. With the knowledge of the LBS positions angles, delays and phases can be updated according to positions of the UE in the snapshots. This technique was introduced in SCME [10] but was not migrated to the later WINNER branches. Assigning different propagation scenarios to different segments is an important 


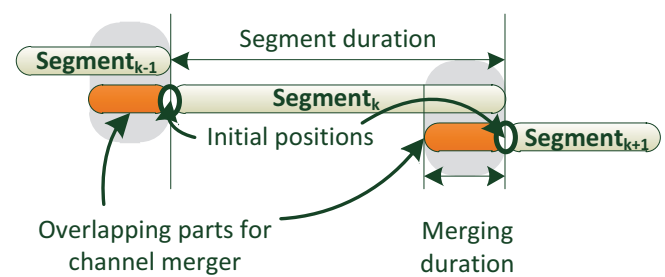

Fig. 5. Overlapping segments can be blended defining a merging duration in which the segments cross fade.

feature of QuaDRiGa. Therefore, a continuous evolution of the channel is realized by smooth transitions between segments (see [2]). This is achieved by blending overlapping parts of the segments as illustrated in Fig. 5. Even if the propagation scenarios between adjacent segments are equal, LSPs will vary which yields to fading of the channel in one segment to another.

\section{F. Interpolating the Coefficients for Varying Speeds}

When the channel sampling theorem is fulfilled, i.e. when there are at least two samples per half wave length, then it is possible to interpolate the channel coefficients to any desired speed and also emulate track segments where the UE accelerates or slows down. Each sample point in time (given in units of seconds) has a corresponding sample point on the user track (in units of meters). For each entry in the movement profile, the channel coefficients are derived using linear interpolation of the path delays, amplitudes and phases between two neighboring values.

\section{VARIABLE COMPLEXITY}

A motivation to address different complexity levels is that not all investigated aspects of future mobile networks require the full complexity of a spatial channel model. Lower complexity of channel modeling leads to shorter optimization cycles especially when simulations for self organizing networks are considered. Also if results from different complexity levels are compared the same basis is maintained. This section gives an example how possible complexity degrees could look like. Referring to QuaDRiGa variable complexity levels can be distinguished as:

1) SISO transmission on power basis excluding fast fading

2) MIMO transmission on LOS basis (single tap)

3) MIMO transmission including fast fading effects and multi-path propagation

The first complexity level is simplified to a received power based SISO channel modeling taking large-scale effects such as antenna directivity, path loss and SF along with the transmit power into account. Due to the increased spatial reuse of radio resources when deploying massively small cells into the standard macro cellular deployment handovers will occur more frequently. Since handover optimization can be based on received power levels from the serving cell and the neighborhood this complexity level is favorable.
The next complexity level is to consider multiple antennas at transmitter and receiver for the investigation of beamforming and polarization effects for LOS connections. The single tap analysis provides a convenient way to conduct radio resource management on a wide band basis with slightly increased computation effort.

The highest complexity level, considering all features QuaDRiGa offers, enables investigations like CoMP, MIMO techniques and frequency selective scheduling with a continuous time evolution not being restrained to short observation periods.

\section{Channel Characteristics}

This section contains the channel characteristics of an NLOS urban macro (WINNER+ C2) scenario of a typical homogeneous triple sectorized hexagonal grid with an intersite distance of 500 meters, as shown beside the geometry curve in 6(a), with directive antennas. Exemplary, the first seven cells were evaluated to provide a possible comparison to characteristics generated with other channel models. In a wireless channel the signal $x$ is transmitted over the channel $H$ with an additional noise $n . y$ is the received signal.

$$
y=H x+n
$$

The received power $P$ at time $\tau$ is therefore given by the squared magnitude of the channel coefficients.

$$
P(\tau)=|H(\tau)|^{2}
$$

The geometry GF is the the expectation value over time and frequency of the power P of the strongest base station of the $j$ base stations in relation to the others (see Fig. 6(a) for the GF of the deployment depicted beside the GF curve). The merit of this metric is to average all fluctuation of the channel over frequency $f$ and time $t$. Hence, this yields only the geometric influences (antenna directivity and path loss).

$$
\mathrm{GF}=10 \log _{10} \frac{E_{t, f}\left[\max P_{j}\right]}{\sum_{j} E_{t, f}\left[P_{j}\right]-E_{t, f}\left[\max P_{j}\right]}
$$

Another characteristic of a channel is the the rms DS. The rms DS provides a measure for the duration in which the rms average of the power $P(\tau)$ arrives at the receiver in relation to earliest significant multi path component. The rms DS includes the mean delay $\bar{\tau}$ :

$$
\bar{\tau}=\frac{\int_{0}^{\infty} \tau P(\tau) d \tau}{\int_{0}^{\infty} P(\tau) d \tau} .
$$

The rms DS $\tau_{\text {rms }}$ is determined according to [11]. The distribution of the the rms DS per sector for an urban macro environment is contained in Fig. 6(c) determined by:

$$
\tau_{\text {rms }}=\sqrt{\frac{\int_{0}^{\infty}(\tau-\bar{\tau})^{2} P(\tau) d \tau}{\int_{0}^{\infty} P(\tau) d \tau}} .
$$

The condition number $\kappa$ of the channel matrix $H$ indicates spatial multiplexing capabilities of the channel. The smaller $\kappa$ is the better $H$ is conditioned. Generally speaking a better 


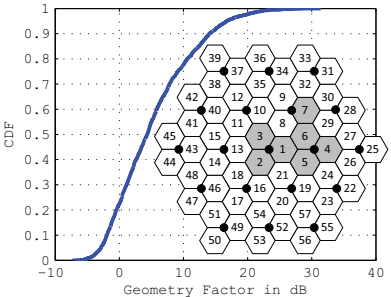

(a) geometry

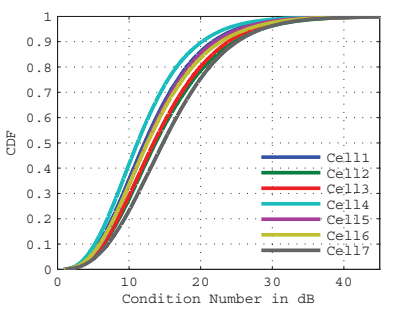

(c) condition number

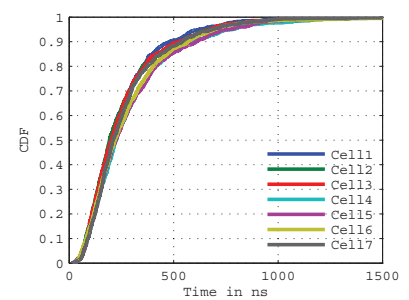

(b) rms DS

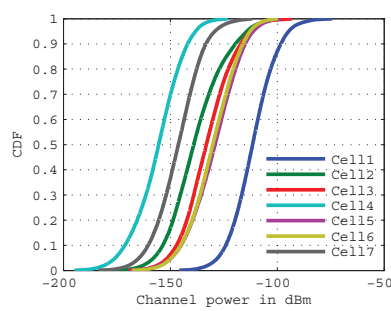

(d) top- $\mathrm{N}$
Fig. 6. Channel characteristics based on a urban macro channel with a typical homogeneous deployment as shown in (a) for co-polarized antennas

conditioning of the channel leads to a higher channel capacity. Fig. 6(c) shows the distribution of the condition number per sector for the priorly mentioned deployment.

$$
\kappa(H)=20 \log _{10}\left|\frac{\lambda_{\max }(H)}{\lambda_{\min }(H)}\right|
$$

The top-N characteristic is a received power based distribution for the users in the center cell. The cumulative distribution function for each sector is shown in Fig. 6(d). The distance of the received power power distribution is an indicator for the strength of interference of the surrounding cells. The received power distributions for the co-located sectors (cell 2 and 3) show almost the same distribution as it should be expected.

\section{Conclusion}

QuaDRiGa provides the required features for the simulation of future mobile networks. It also enables different degrees of complexities according to the intended investigated aspects of the network. Fig. 6 shows that QuaDRiGa also provides the well know channel characteristics for typical macro cellular deployments. The feature of continuous time evolution is an essential feature for simulations with long observation periods which cannot be simulated to the best of our knowledge with a current open source channel model. It combines features like transition between different propagation scenarios, polarimetric antenna model and spatially correlated LSP maps. QuaDRiGa provides a holistic modeling approach for performance evaluation of future heterogeneous radio networks. Beside transmission concepts or radio resource management schemes, also self organizing networks - but with a lower modeling complexity - can be investigated.

\section{ACKNOWLEDGMENT}

The authors are grateful for financial support from the German Ministry of Economics (BMWi) in the national collabo- rative project IntelliSpektrum under contract No. 01ME11024.

\section{REFERENCES}

[1] Technical Specification Group Radio Access Network, "Spatial channel model for multiple input multiple output (mimo) simulations," 3rd Generation Partnership Project (3GPP), TR 25.996, Tech. Rep. V6.1.0, 2003.

[2] P. Kyösti et al., "WINNER II Channel Models," IST-4-027756 WINNER II D1.1.2 V1.1, Sep. 2007. [Online]. Available: https://www.istwinner.org/WINNER2-Deliverables/D1.1.2v1.1.pdf

[3] J. Meinilä, P. Kyösti, L. Hentilä, T. Jämsä, E. Suikkanen, E. Kunnari, and M. Narandzic, "D5.3: WINNER+ Final Channel Models," CELTIC CP5-026 WINNER+, Jun. 2010.

[4] M. Gudmundson, "Correlation model for shadow fading in mobile radio systems," Electronics Letters, vol. 27, no. 23, pp. 2145 -2146, nov. 1991.

[5] K. Zayana and B. Guisnet, "Measurements and modelisation of shadowing cross-correlations between two base-stations," in Universal Personal Communications, 1998. ICUPC '98. IEEE 1998 International Conference on, vol. 1, oct 1998, pp. $101-105$ vol.1.

[6] L. Thiele, T. Wirth, K. Börner, M. Olbrich, V. Jungnickel, J. Rumold and S. Fritze, "Modeling of 3D Field Patterns of Downtilted Antennas and Their Impact on Cellular Systems," in International ITG Workshop on Smart Antennas (WSA 2009), Berlin, Germany, Feb. 2009.

[7] Technical Specification Group Radio Access Network, "Further advancements for E-UTRA physical layer aspects," 3rd Generation Partnership Project (3GPP), TR 36.814, Tech. Rep. V9.0.0, 2010.

[8] L. Jiang, L. Thiele, and V. Jungnickel, "On the Modelling of Polarized MIMO Channel," in 13th European Wireless Conference, Paris, France, Apr. 2007.

[9] S. Jaeckel, K. Börner, L. Thiele, and V. Jungnickel, "A Geometric Polarization Rotation Model for the 3D Spatial Channel Model," IEEE Transactions on Antennas and Propagation, pp. 1-11, 2012.

[10] D. Baum, J. Hansen, and J. Salo, "An interim channel model for beyond-3g systems: extending the 3gpp spatial channel model ( $\mathrm{scm})$," in Vehicular Technology Conference, 2005. VTC 2005-Spring. 2005 IEEE 61st, vol. 5, may-1 june 2005, pp. 3132 - 3136 Vol. 5 .

[11] A. Goldsmith, Wireless Communications. New York, NY, USA Cambridge University Press, 2005. 